\title{
Laser diodes in holographic optical tweezers
}

\author{
Sławomir Drobczyński, ${ }^{* 1}$ Jan Masajada, ${ }^{1}$ Marta Woźniak ${ }^{2}$ and Piotr Ziółkowski ${ }^{2}$ \\ ${ }^{1}$ Institute of Physics, Wrocław University of Technology, Wyb. Wyspiańskiego27, 50-370 Wrocław, \\ ${ }^{2}$ Department of Pathology, Faculty of Medicine, Wroctaw Medical University, Marcinkowskiego 1,50-368 Wroctaw
}

Received September 11, 2013; accepted February 03, 2014; published March 31, 2014

\begin{abstract}
Laser diodes are attractive sources of laser radiation in terms of price, size, controllability and availability of a wide spectrum of light emitted wavelengths. In this paper we present the use of three types of laser diodes in the holographic optical tweezers. We showed that they can be applied for effective trapping and live cells manipulation, despite their relatively small power.
\end{abstract}

Most popular light sources used in optical tweezers [1-2] are Nd: YAG, or argon-ion lasers. Replacing them by laser diodes would reduce the costs and size of optical tweezers. Before that happens, the typical drawbacks of laser diodes must be overcome. These drawbacks are: small beam power, elliptical beam profiles, large far-field divergence angles. For these reasons, the laser diodes must be used with additional optics for collimation and laser beam geometry correction.

Various applications require lasers with various wavelengths. A strongly focused laser beam can destroy the biological structures in an optical trap. Water exhibits strong absorption in the infrared region while most of the biological samples have strong absorption in the ultraviolet and visible regions. For this reason, optical tweezers designed for biomedical research use lasers working in the near infrared range. Optical tweezers systems are more and more often integrated with an additional module as, for example, a fluorescence module. In such a case the compact design of the measurement system is possible by the use of single laser diodes for both trapping and sample stimulating. Many users are able to buy a set of relatively cheap laser diodes with different wavelengths and the laser mount can be designed in a way enabling easy source change.

The use of laser diodes in optical tweezers has been already reported in some papers. In paper [3-5] the optical tweezers based on a biological microscope and equipped with a single mode laser diode was reported. The laser beam was collimated by an aspherical lens and the elliptical beam profile was corrected by an anamorphic prism pair. The system enabled single trap generation, the position of which was controlled by microscopic table movement or laser beam deflection. In paper [6] the authors use a single-mode laser diode in holographic optical tweezers. The holographic technique

\footnotetext{
*E-mail: slawomir.drobczynski@pwr.wroc.pl
}

enables the generation of few tens of traps. Moreover, the traps of different kind (light, dark [7], Bessel [8]) can be generated simultaneously. The great advantage of holographic optical tweezers is that the position of the traps can be controlled independently in the $x-y-z$ direction purely optically, i.e. just by displaying subsequent holograms on a spatial light modulator. On the other hand, the quality of a laser source is more significant for holographic optical tweezers, so applying lasers diodes is a more difficult task.

In this paper we test the holographic optical tweezers system with a single-mode laser diode, multimode laser diode and single-mode laser diode coupled to an optical fiber. We showed that effective optical trapping for each of these three types of sources is realizable. The tests were performed with a sample containing viable tumor cells. The diodes used in our experiments had the following parameters: a) single mode diode (SMD) $\lambda=980 \mathrm{~nm}$, $\mathrm{P}_{\max }=300 \mathrm{~mW}, \quad 30^{\circ} \perp, \quad 7^{\circ} \|, \quad 1 \times 3 \mu \mathrm{m}$ emitter size; b) multimode diode $\lambda=808 \mathrm{~nm}, \mathrm{P}_{\max }=1 \mathrm{~W}, 35^{\circ} \perp, 3^{\circ} \|$, $1 \times 100 \mu \mathrm{m}$; c) SMD $\lambda=980 \mathrm{~nm}, \mathrm{P}_{\max }=450 \mathrm{~mW}$, single mode fiber pigtail NA=0.14 (MFD, mode field diameter) $5.9 \mu \mathrm{m}$ with fiber Bragg grating frequency stabilization.

The optical system of our holographic tweezers was designed for achromatic light and a circularly symmetrical optical beam. So to improve its performance the diodes were supported by an aspherical correction lens. Unfortunately, this lens did not correct beam astigmatism in a satisfactory way. Laser diode coupling to an optical fiber resulted in the best circularly symmetrical output beam. Moreover, the use of a standard fiber optic connector such as FC greatly facilitates the adjustment of the system. Figure 1 shows the focused spot, at the focal plane, of the focusing objective for a multimode diode, single mode diode and pigtail single mode diode, respectively. These spots were measured directly in the holographic optical tweezers. 

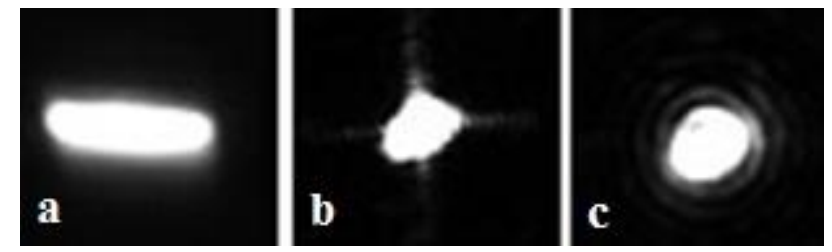

Fig. 1. Focused light spots: a) multi mode diode; b) single mode diode; c) pigtail single mode diode.

All the laser diodes were powered by constant current configuration, and the junction temperature was stabilized by the Peltier element. This protects the system against spectral instability, which may result from the changes in the length of a resonator due to temperature variations. An important problem was the mechanical stabilization of an optical fiber in the case of a pigtail diode. Even relatively small mechanical stress changes the state of output light polarization. Maintaining the state of polarization of emitted light is essential for the effective reconstruction of computer-generated holograms (CGH). The fluctuations in the laser beam power result in the fluctuation of optical trap stiffness. The applied method for laser diodes stabilization was sufficient. The variations in the optical trap stiffness "were lost" in the system electronic noise.

The value of optical trap stiffness was measured as a function of laser power. For this purpose the standard method was used [9]. Linear dependence was obtained, which is in agreement with the theory and studies reported by other authors [10]. When the diode current was maximal, for the $3 \mu \mathrm{m}$ polystyrene microsphere $n=1.6$, the following values for trap stiffness were obtained (where the laser power was measured before the focusing microscopic objective): a) multi mode diode: $808 \mathrm{~nm}$, $20 \mathrm{~mW}, 1.8 \cdot 10^{-6} \mathrm{~N} / \mathrm{m}$; b) single mode diode: $980 \mathrm{~nm}, 18$ $\mathrm{mW}, 5.64 \cdot 10^{-6} \mathrm{~N} / \mathrm{m}$; c) pigtail single mode diode: $980 \mathrm{~nm}$, $140 \mathrm{~mW}, 9.54 \cdot 10^{-6} \mathrm{~N} / \mathrm{m}$.

Working in the IR range results in large power losses. In the holographic optical tweezers this loss is due to the absorption by microscopic objectives and limited diffraction efficiency of a spatial light modulator (Hamamatsu X10468-07). The biggest impact on diffractive structure efficiency have the SLM fill factor (95\%) and phase matching levels for a given wavelength [11]. When using multi-mode diodes the specific emitter geometry causes additional problems in this matter. Thus the number of effective traps generated with diodes is even five times smaller as compared to the system with YAG or other high power lasers.

Our system was equipped with a touch screen. The trapped objects were moved just by pointing them with a finger and moving the finger to the desired position [12]. The monitor displayed an image of the sample in microscopic white lamp illumination. The traps position and geometry was controlled by real time holograms recomputing. The holograms patterns were sent one by one to a spatial light modulator. To ensure a sufficiently high computation speed, specialized libraries, i.e. Computing Intel ${ }^{\circledR}$ Integrated Performance Primitives (Intel ${ }^{\circledR}$ IPP) optimized for Intel processors, were used. The surface of the SLM available on the market is not flat. We used the phase correction map provided by the SLM manufacturer. Thus the pattern displayed on the SLM was the superposition of two terms: gratings or holograms for optical trap generation and positioning and a phase map for surface curvature correction. Figure 2 shows the Laguerre-Gauss beam with topological charge 6 (dark trap) generated with uncorrected (a) and corrected (b) pattern displayed on the SLM.
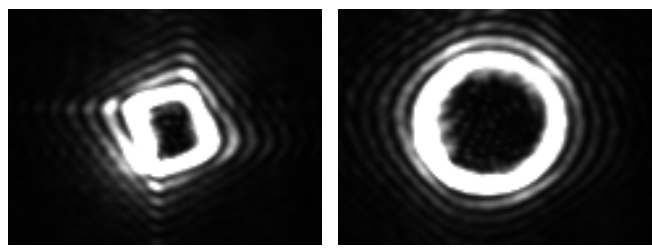

Fig. 2. Laguerre-Gauss beam. Left: non-corrected. Right: corrected.

We have examined two methods for generating and handling traps: using Fourier holograms and phase-blazed diffraction gratings [13]. Fourier holograms were optimized by applying the iterative Gerchberg-Saxton algorithm [14]. This iterative algorithm did not improve the diffraction efficiency in a significant way [15], but the multimode diode beam symmetry was improved (Fig. 3).

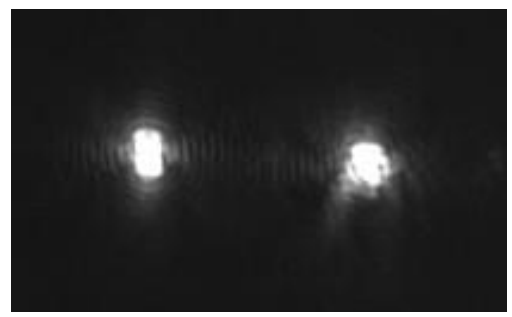

Fig. 3. Focused light spots. Left: generated by blazed diffraction phase-grating. Right: generated by a hologram calculated with Gerchberg-Saxton algorithm (after 6 iterations).

Improving the shape of the focal spot can also be achieved by reducing the aperture of the hologram (Fig. 4). Note, however, that by reducing the aperture of the beam the intensity gradient due to diffraction effects is also reduced. This results in the drop of trap stiffness. On the other hand, the outer part of the structure (beyond the defined circular aperture) can be used for additional tasks. For example, the influence of the zero order beam can be reduced. The zero order beam creates an additional trap which position and parameters cannot be controlled. We can define a Fresnel structure which moves the focal plane 
of the zero order beam deeper into the sample volume. The other solution displays the random phase pattern (Fig. 4a). As a result, the light intensity of the zero order beam becomes lower.
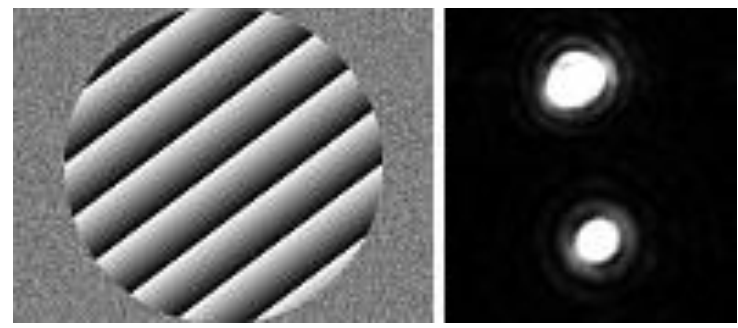

Fig. 4. Left: hologram with a circular aperture. The random phase pattern is displayed beyond the hologram aperture. Right: light spot without an aperture and more symmetric generated with an aperture.

We have tested two types of immersion microscopic objectives. The Olympus objective with $\mathrm{NA}=1.2,60 \times$ was not sufficient for stable trapping of objects below $5 \mu \mathrm{m}$ when using a multi mode diode. With the Olympus objective NA=1.3, 100 $\times$ the Polystyrene beads (Polybead ( $)$ Polystyrene Microsphere) with a diameter of $3 \mu \mathrm{m}$ were successfully trapped. In Ref. 16 the authors study wavefront corrections for all subsequent elements in the holographic tweezers system. Thus, it should be possible to trap even smaller particles with a laser diode.

Our tests were carried out in the liquid sample containing both viable cancer cells SW 620 (Fig. 5) and polystyrene beads. The cells were manipulated by pushing them with the beads. For all the three diodes used in the experiments the trap stiffness was high enough to manipulate living cells via trapped polystyrene beads ( $3 \mu \mathrm{m}$ and $5 \mu \mathrm{m}$ in diameter and objective NA=1.3, 100×). We used two or three independent traps for this purpose. The cells were moved in all the three directions $\mathrm{x}-\mathrm{y}-\mathrm{z}$. The $\mathrm{z}$-shift was obtained by changing the optical power of the Fresnel lens.

In conclusions, we claim that laser diodes can be used for carrying out experiments with optical trapping. The number of traps which can be effectively generated is limited when compared with a system equipped with high power laser sources, but the diodes are cheap. The user may have a large selection of diodes emitting light of different wavelengths. The laser port can be built in a way which enables fast diode changing. Thus the single and relatively cheap optical tweezers can be applied for a wide range of experiments. Certainly, there are experiments which cannot be performed in this way. For example, the evaluation of living cells mechanical properties requires strong trapping forces which are not possible with laser diodes. But still the amount of possible research work is very large. For example, we have performed tests on cancer cells surviving in various solutions, which is necessary for measuring their electrical polarizability. The tests were successfully carried out during many hours.

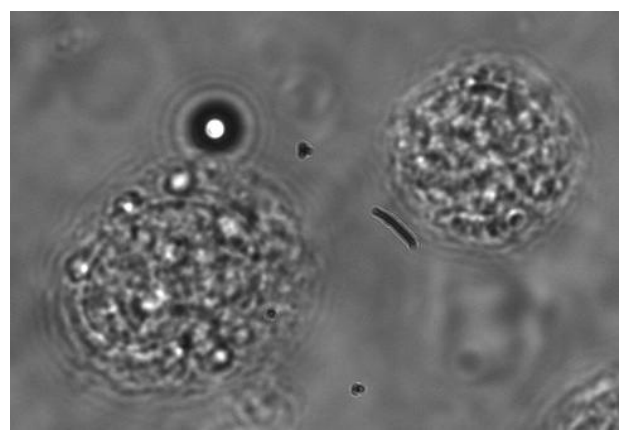

Fig. 5. Colorectal adenocarcinoma, human colon cancer SW620 cell line and $3 \mu \mathrm{m}$ polystyrene bead.

Our results show that applying a laser diode in holographic optical tweezers in the IR range makes sense. Still there is room for improving their performance. Moreover, the diode technology is progressing very fast. The prices are dropping and parameters become better. Having all these factors in mind, we believe that laser diodes will be more and more often used in holographic optical tweezers.

This work was supported by the Polish Ministry of Scientific Research and Information Technology under Grant No. N N518 498839

\section{References}

[1] D. Stevenson, F. Gunn-Moore, K. Dholakia, J. Biomedical Optics 15(4), 041503-1-041503-21 (2010).

[2] K. Neuman, S. Blocka, Rev. Sci. Instrum. 75 (9), 2787 (2004).

[3] R. Afzal, E. B. Treacy, Rev. Sci. Instrum. 63 (4), 2157 (1992).

[4] Z. Ulanowski, I. Ludlow, Meas. Sci. Technol. 11, 1778 (2000).

[5] M. Sery, Z. Lostak, M. Kalman, P. Jakl, P. Zemanek, Proc. SPIE 6609, 66090N-1 (2007)

[6] R.L. Eriksen, V. Ricardo, J. Glückstad, Opt. Expr. 10 (14), 597 (2002).

[7] D. Cojoc, V. Garbin, E. Ferrari, L. Businaro, F. Romanato, E. Di Fabrizio, Microelectr. Eng. 78-79, 125 (2005).

[8] D. McGloin, K. Dholakia, Cont. Phys. 46(1), 15 (2005).

[9] K. Berg-Sørensen, H. Flyvbjerg, Rev. Sci. Instrum. 75(3), 594 (2004).

[10] W. Singer, S. Bernet, N. Hecker, M. Ritsch-Marte, J. Modern Optics 47 (14/15), 2921 (2000).

[11] Ch.H.J. Schmitz, J.P. Spatz, J.E. Curtis, Opt. Expr. 13 (21), 8678 (2005).

[12] R.W. Bowman, G. Gibson, D Carberry, L. Picco, M. Miles, M.J. Padgett, J. Opt. 13, 044002 (2011).

[13] J. Liesener, M. Reicherter, T. Haist, H.J. Tiziani, Opt. Comm. 185 (13), 77 (2000).

[14] D.G. Grier et al., Rev. Sci. Instrum. 72 (3), 1810 (2001).

[15] J.E. Curtis, Ch.H.J. Schmitz, J.P. Spatz, Opt. Expr. 30(16), 2086 (2005).

[16] T. Cizmar, M. Mazilu, K. Dholakia, Nature Phot. 4, 388 (2010). 